\title{
Epidemiology of gastric cancer: global trends, risk factors and prevention
}

\author{
Prashanth Rawla ${ }^{1}$, Adam Barsouk ${ }^{2}$ \\ ${ }^{1}$ Department of Internal Medicine, SOVAH Health, Hospitalist, Martinsville, USA \\ ${ }^{2}$ Hillman Cancer Center, University of Pittsburgh, PA, USA
}

Gastroenterology Rev 2019; 14 (1): 26-38

DOI: https://doi.org/10.5114/pg.2018.80001

Key words: stomach cancer, epidemiology, risk factors, prevention, gastric cancer, incidence, mortality, survival.

Address for correspondence: Prashanth Rawla, Department of Internal Medicine/Hospitalist, SOVAH Health, Martinsville, VA, 24112 USA,

phone: 732-982-7357, e-mail: rawlap@gmail.com

\begin{abstract}
Gastric cancer remains one of the most common and deadly cancers worldwide, especially among older males. Based on GLOBOCAN 2018 data, stomach cancer is the $5^{\text {th }}$ most common neoplasm and the $3^{\text {rd }}$ most deadly cancer, with an estimated 783,000 deaths in 2018. Gastric cancer incidence and mortality are highly variable by region and highly dependent on diet and Helicobacter pylori infection. While strides in preventing and treating $H$. pylori infection have decreased the overall incidence of gastric cancer, they have also contributed to an increase in the incidence of cardia gastric cancer, a rare subtype of the neoplasm that has grown 7 -fold in the past decades. A better understanding of the etiology and risk factors of the disease can help reach a consensus in approaching $H$. pylori infection. Dietary modification, smoking cessation, and exercise hold promise in preventing gastric cancer, while genetic testing is enabling earlier diagnosis and thus greater survival.
\end{abstract}

\section{Introduction}

Although it is steadily declining in incidence, cancer of the stomach (also known as gastric cancer) remains one of the most common and deadly neoplasms in the world [1]. The stomach, located in the digestive tract between the esophagus and small intestine, secretes enzymes and gastric acid to aid in the digestion of food. It also secretes the intrinsic factor necessary for the absorption of vitamin $B_{12}$. The stomach is lined with a mucous membrane composed of columnar epithelial cells and glands. These cells are prone to inflammation, known as gastritis, which can lead to peptic ulcers, and ultimately, gastric cancer [2].

Gastric adenocarcinomas are primarily classified as cardia and non-cardia based on their anatomic site. Cancers of the gastric cardia arise in the region adjoining the esophageal-gastric junction and thus share epidemiological characteristics with esophageal adenocarcinoma (EAC). Non-cardia cancer, also known as distal stomach cancer, is more common and arises in the lower portion of the stomach [3]. Histologically the two main types of gastric cancer are the diffuse and intestinal type [4].
According to GLOBOCAN 2018 data, gastric cancer is the third leading cause of cancer deaths worldwide, following only lung and colorectal cancer in overall mortality. About 1 in 12 of all oncological deaths are attributable to gastric cancer. Gastric cancer has the fifth highest incidence among cancers, with $5.7 \%$ of all new cases attributable to the disease. Over a million new cases of gastric cancer are diagnosed, worldwide, each year [1, 5]. Gastric cancer is also one of the most behaviorally influenced, and thus preventable, of major cancers [2].

\section{Epidemiology \\ Incidence}

Over one million cases of gastric cancer are diagnosed each year around the world. Stomach cancer is the $5^{\text {th }}$ most commonly diagnosed cancer in the world, and the $7^{\text {th }}$ most prevalent (Figure 1). The cumulative risk of developing gastric cancer from birth to age 74 is $1.87 \%$ in males and $0.79 \%$ in females worldwide [1].

Gastric cancer is more prevalent in males. In developed countries, gastric cancer is 2.2 times more likely to be diagnosed in males than females. In developing countries, this ratio is 1.83 . In 5 countries worldwide, 
gastric cancer has the highest incidence among all cancers for males. There is no country where it is the most diagnosed cancer in females [1].

Cancers of the stomach are more frequently diagnosed in developed nations. The average incidence rate among high-middle Human Development Index (HDI) nations is 20 per 100,000 for males, while the average rate among low-middle HDI nations is 6.6 per 100,000 [1].
The incidence of gastric cancer is highly variable by region and culture. Incidence rates are highest in Eastern and Central Asia and Latin America (Figure 2) [6]. In East Asia, the average incidence of gastric cancer is 32.1 per 100,000 among males and 13.2 among females. In North America, this incidence is 5.6 per 100,000 . The rate is lowest in North and East Africa, with only 4.7 annual diagnoses per 100,000 males. The Republic of

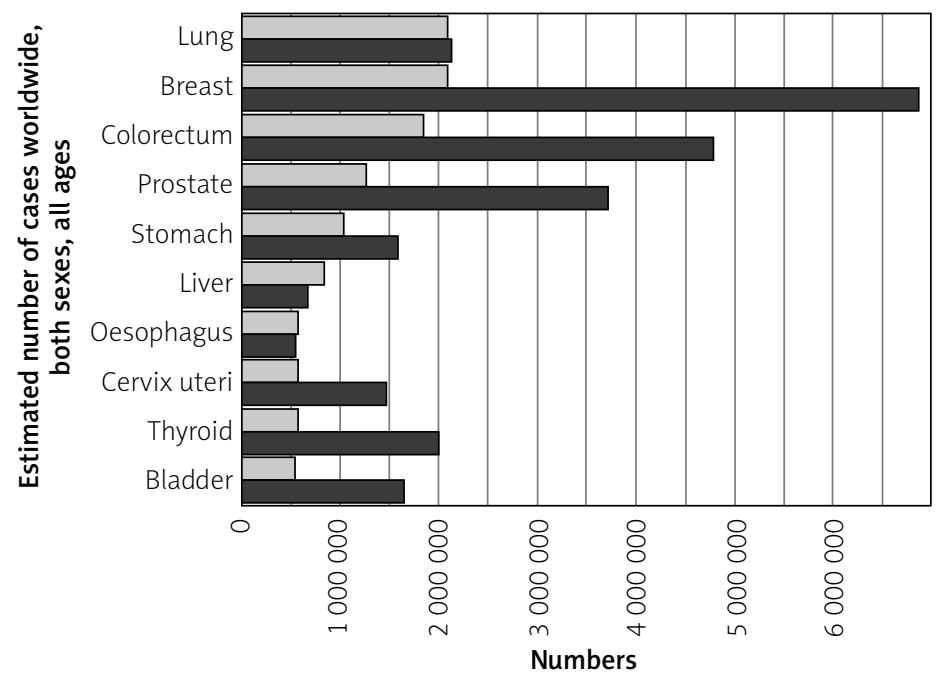

$\square$ Incidence $\quad \square$ Prevalence

Figure 1. Graph shows the estimated incidence and prevalence of cancer cases worldwide, both sexes, all ages (reproduced from http://globocan.iarc.fr/ [5])

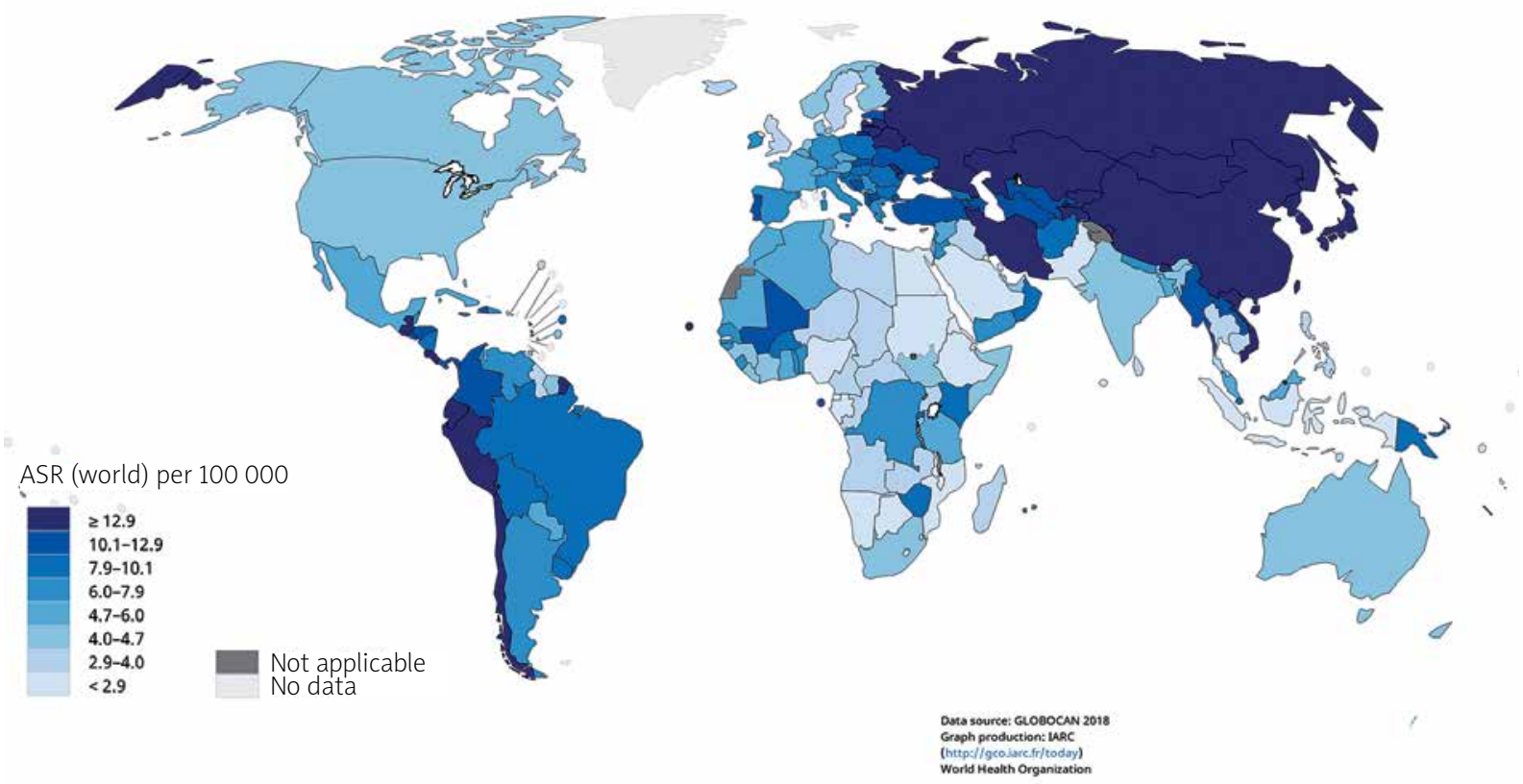

Figure 2. Map shows the estimated age-standardized incidence rates (world) for stomach cancer in 2018, both sexes, all ages (reproduced from http://globocan.iarc.fr/ [5]) 
Korea has the highest national incidence with almost 60 per 100,000 new cases annually for males. While female incidence rates are lower (only 25 per 100,000 in Korea); they follow the same regional trend (Figures $3,4)[1,5]$.

Although declining in incidence, non-cardia stomach cancer continues to be diagnosed twice as often as cardia [2].

\section{Mortality}

Gastric cancer was the most common cause of cancer-related mortality globally up to the mid-1990s. Gastric cancer accounts for 783,000 deaths each year, making it the third most deadly cancer among males worldwide (Figure 5). 8.3\% of all cancer deaths are attributable to gastric cancer. The cumulative risk of death

A

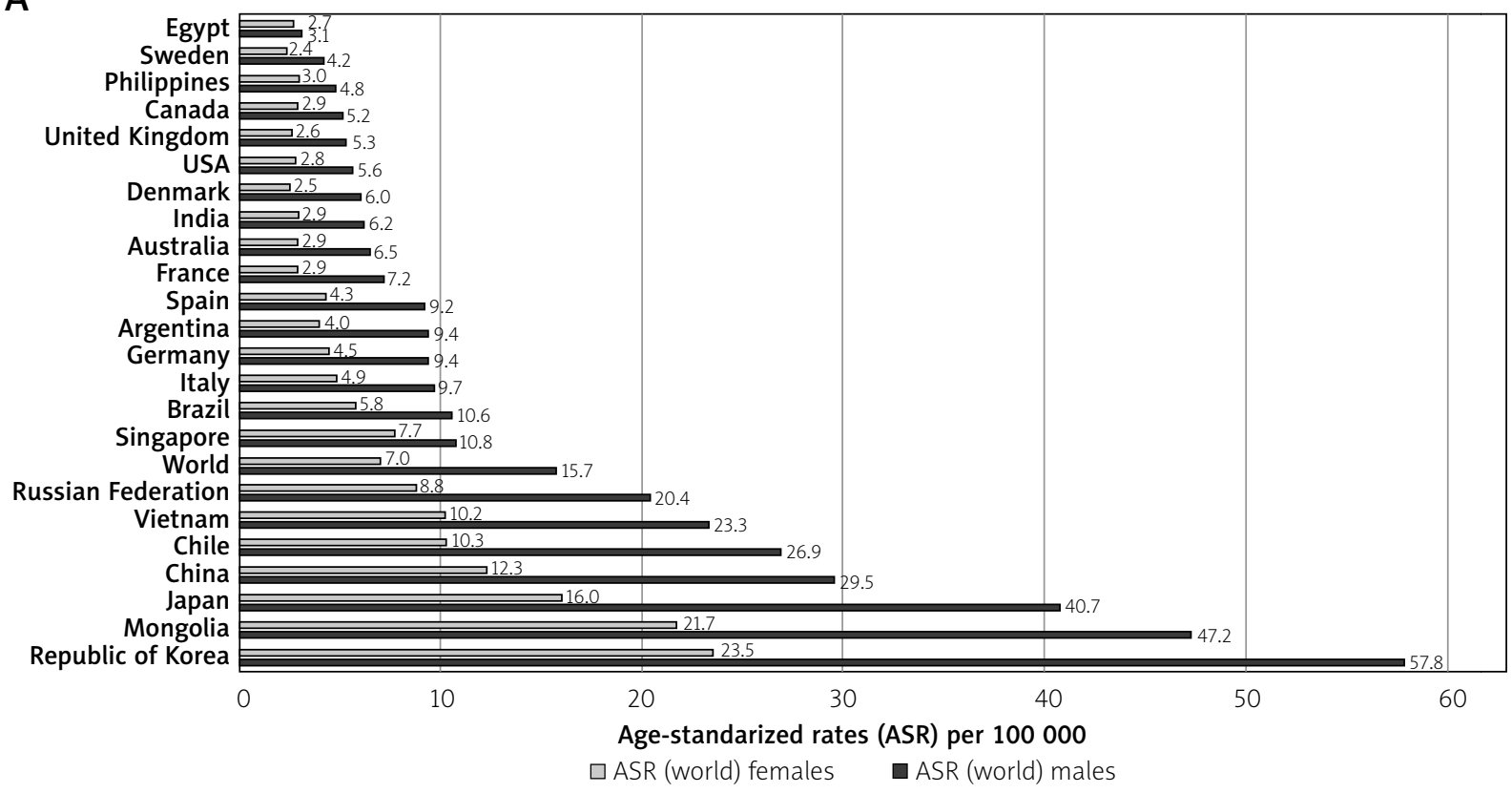

B

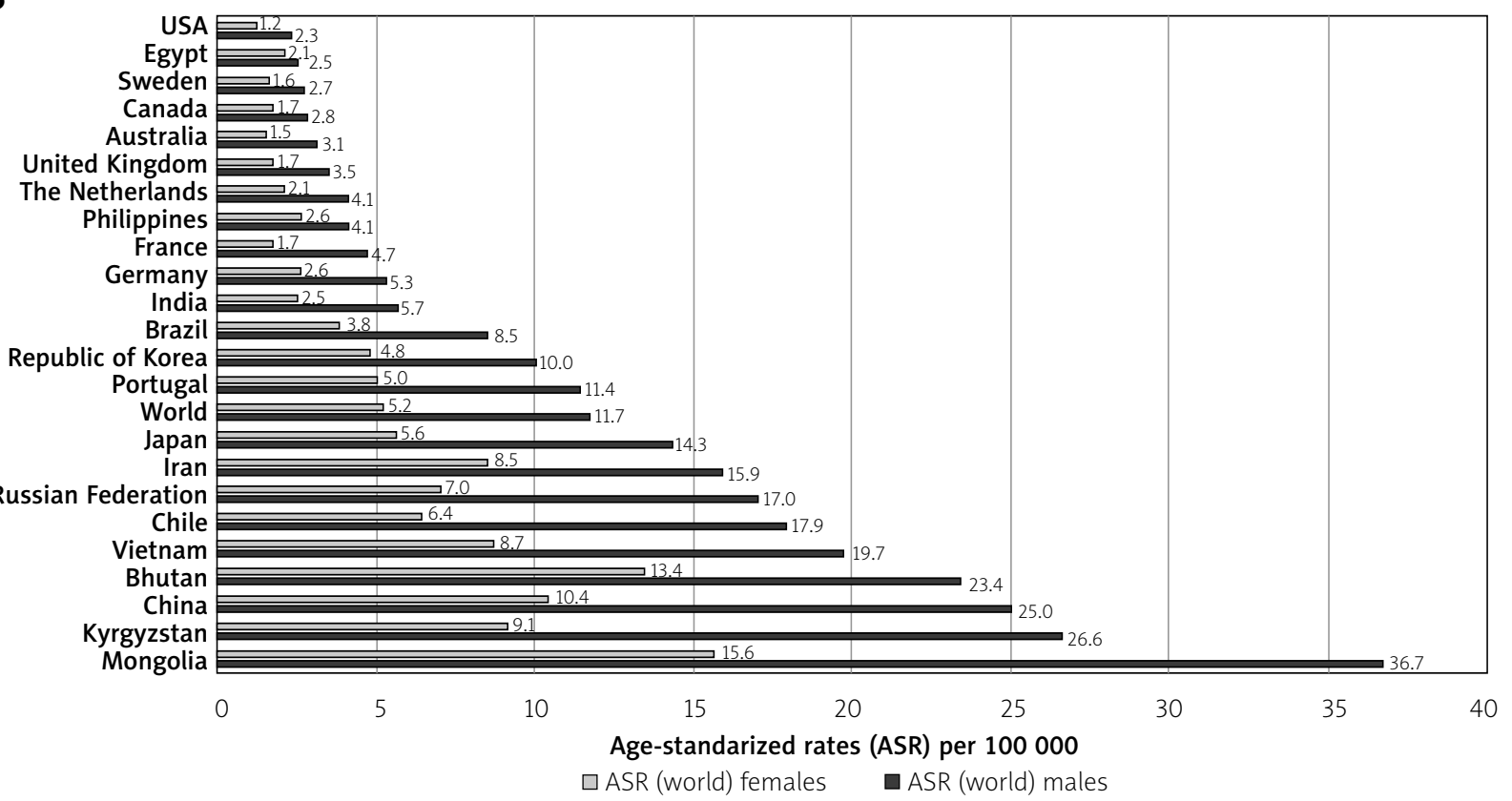

Figure 3. A - Estimated age-standardized incidence rates (World) in 2018, stomach, males and females, all ages (reproduced from http://globocan.iarc.fr/ [5]). B - Estimated age-standardized mortality rates (World) in 2018, stomach, males and females, all ages (reproduced from http://globocan.iarc.fr/ [5]) 


\begin{tabular}{|c|c|c|}
\hline \multicolumn{3}{|c|}{ Both sexes } \\
\hline Rank & Country & $\begin{array}{l}\text { Age-standardised } \\
\text { rate per } 100,000\end{array}$ \\
\hline 1 & $\begin{array}{l}\text { South } \\
\text { Korea }\end{array}$ & 39.6 \\
\hline 2 & Mongolia & 33.1 \\
\hline 3 & Japan & 27.5 \\
\hline 4 & China & 20.7 \\
\hline 5 & Bhutan & 19.4 \\
\hline 6 & Kyrgyzstan & 18.6 \\
\hline 7 & Chile & 17.8 \\
\hline 8 & Belarus & 16.5 \\
\hline 9 & Peru & 16.1 \\
\hline 10 & Vietnam & 15.9 \\
\hline
\end{tabular}

\begin{tabular}{|c|c|c|}
\hline \multicolumn{3}{|c|}{ Men } \\
\hline Rank & Country & $\begin{array}{l}\text { Age-standardised } \\
\text { rate per } 100,000\end{array}$ \\
\hline 1 & $\begin{array}{l}\text { South } \\
\text { Korea }\end{array}$ & 57.8 \\
\hline 2 & Mongolia & 47.2 \\
\hline 3 & Japan & 40.7 \\
\hline 4 & China & 29.5 \\
\hline 5 & Kyrgyzstan & 29.3 \\
\hline 6 & Chile & 26.9 \\
\hline 7 & Belarus & 25.1 \\
\hline 7 & Cape Verde & 25.1 \\
\hline 9 & Kazakhstan & 24.7 \\
\hline 10 & Bhutan & 24.2 \\
\hline
\end{tabular}

Women
\begin{tabular}{|r|l|r|}
\hline Rank & Country & $\begin{array}{r}\text { Age-standardised } \\
\text { rate per 100,000 }\end{array}$ \\
\hline 1 & $\begin{array}{l}\text { South } \\
\text { Korea }\end{array}$ & 23.5 \\
\hline 2 & Mongolia & 21.7 \\
\hline 3 & Japan & 16 \\
\hline 4 & Guatemala & 14.2 \\
\hline 5 & Bhutan & 13.5 \\
\hline 6 & Peru & 13.4 \\
\hline 7 & Mali & 12.8 \\
\hline \hline 8 & China & 12.3 \\
\hline \hline 9 & Ecuador & 12.2 \\
\hline 10 & Tajikistan & 11.6 \\
\hline
\end{tabular}

Figure 4. Table shows the estimated age-standardized incidence rates per 100,000 for stomach cancer in 2018, all ages, in both sexes, men and women (reproduced and modified from http://globocan.iarc.fr/ [5])
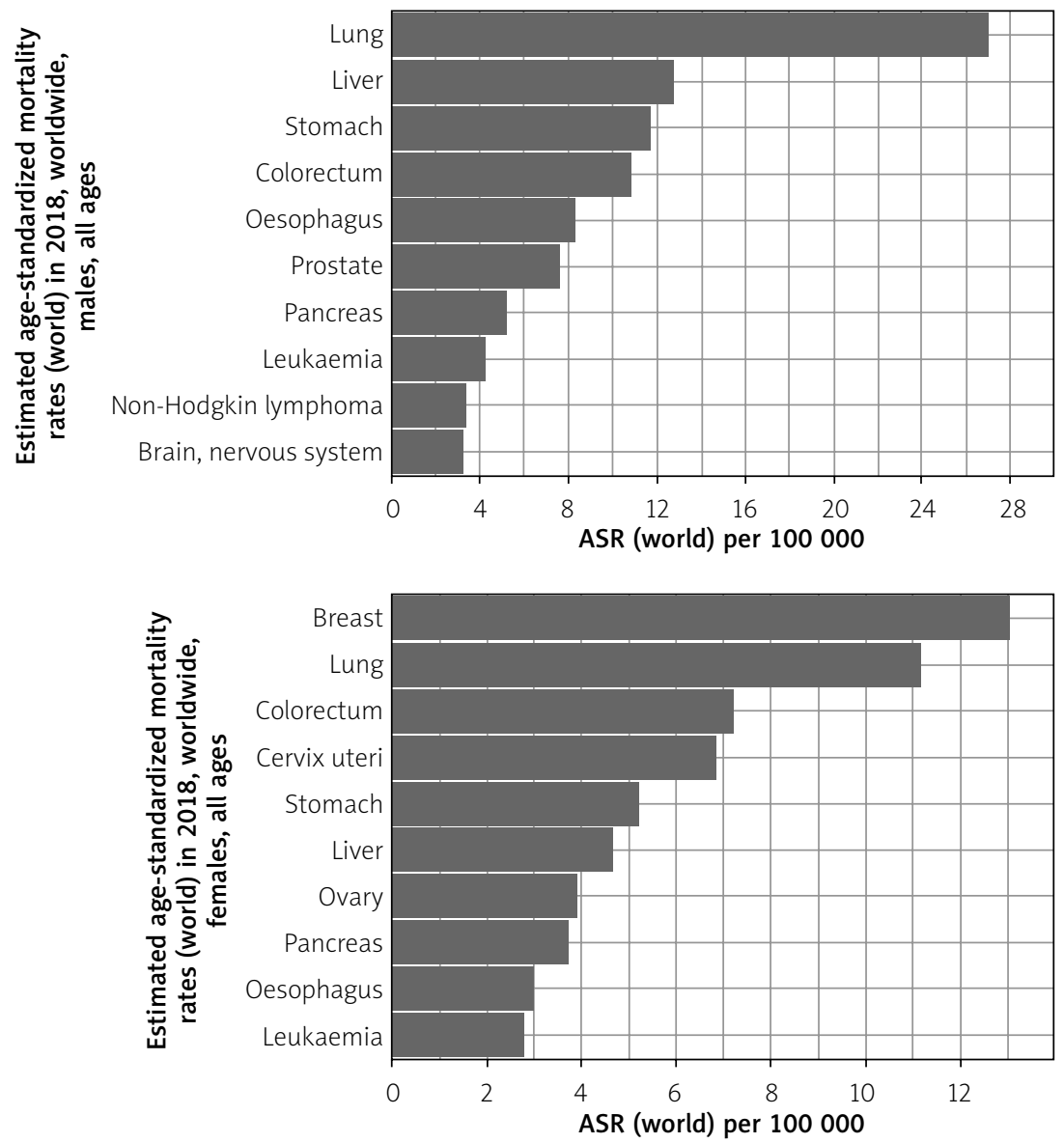

Figure 5. Graph shows the estimated age-standardized mortality rates (world) in 2018, worldwide, males and females, all ages (reproduced from http://globocan.iarc.fr/ [5])

from gastric cancer, from birth to age 74 , is $1.36 \%$ for males and $0.57 \%$ for females [1].

Mortality from gastric cancer is higher among males. Mortality rates are high in eastern and central Asia and Latin America, the same regions with high incidence.
Due to historically low survival rates and few treatment options, especially in developing nations, reducing incidence seems to be the key to reducing mortality [6].

For males, gastric cancer is the leading oncological cause of death in 10 nations worldwide, specifically, 


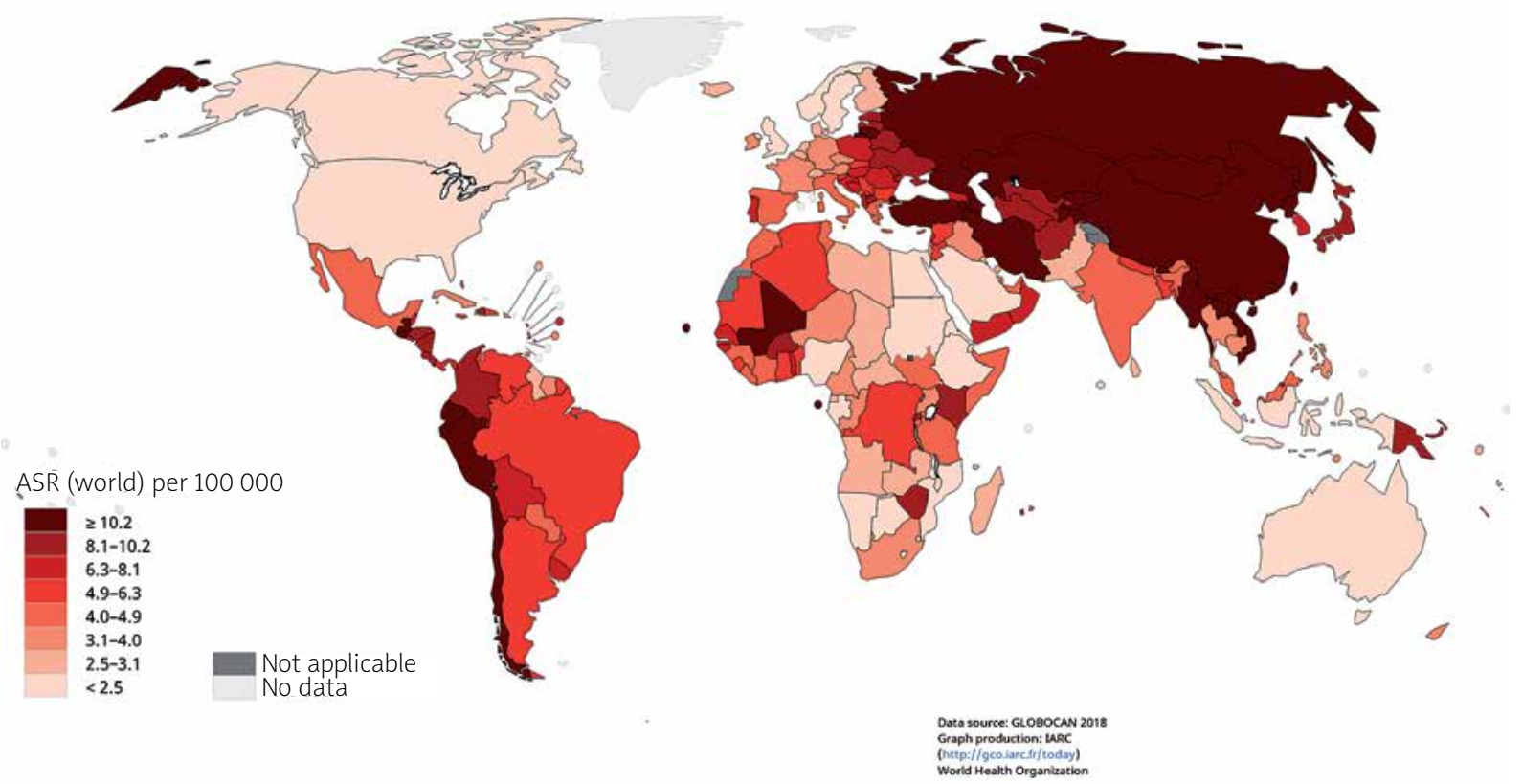

Figure 6. Map shows the estimated age-standardized mortality rates (world) for stomach cancer in 2018, both sexes, all ages (reproduced from http://globocan.iarc.fr/ [5])

eastern and central Asian nations such as Iran, Kyrgyzstan, and Turkmenistan. For females, it is the leading oncological cause of death in 4 nations (Figure 6) [1].

\section{Trends}

The incidence of gastric cancer has steadily declined worldwide over the past 50 years. These declines preceded the successful reduction of $H$. pylori infection, and are likely attributable to changes in food preservation, such as less pickling of vegetables, and less smoking and processing of meat. The decline has also been elicited by the greater availability of fresh fruits and vegetables [6].

The second major factor in gastric cancer decline has been the success in preventing and treating $H$. pylori infections in much of the developing world. As many as $90 \%$ of cases of non-cardia gastric cancer are attributable to $H$. pylori, which explains why the incidence of that subtype of gastric cancer has declined in step with declining infection rates [6]. Meanwhile, during that same period, cardia-subtype gastric cancers have increased 7-fold, especially in the developed world [2].

Non-cardia cancer incidence rates are falling in East Asia due to a reduction of $H$. pylori infections. However, Latin America is not witnessing a similarly effective campaign against $H$. pylori, and gastric cancer incidence is likely to remain high there for the foreseeable future [6].

Gastric cancer survival rates have also steadily improved over the past 40 years thanks to earlier detection and better treatment options [6].

\section{Survival}

The 5 -year survival rate for gastric cancer is 31\% in the United States. Average survival rates reflect the fact that most cases diagnosed are already metastatic. The 5 -year survival rate for pre-metastatic diagnosis is 67\%. Survival is highly variable based on stage during surgical intervention. The 5 -year survival rates for stage IA and IB tumors treated with surgery are $94 \%$ and $88 \%$, respectively. On the other hand, stage IIIC tumors treated with surgery had a 5-year survival rate of $18 \%$ [7].

Asian patients in the United States tend to have a better prognosis than Caucasians. Asians have a $12 \%$ higher 5-year survival rate. They are diagnosed earlier, have more lymph nodes examined, and more of their lymph nodes test positive [8].

In the United Kingdom, the 5-year survival rate is $19 \%$, and the 10 -year survival rate is $15 \%$. Nevertheless, these rates represent a marked improvement in gastric cancer treatment. For the 1971-1972 period in the UK, the 5 -year survival rate was $5.3 \%$, and the 10 -year survival rate was $4.1 \%$ (Figure 7) [9].

The average 5 -year survival rate in Europe is $26 \%$, higher than in the UK but lower than that of the US. The highest survival rate in Europe belongs to Iceland, which reports a 42\% 5-year survival rate among women [9].

\section{Etiology}

Ninety-five percent of cancers of the stomach are adenocarcinomas, followed by primary gastric lympho- 
ma. These adenocarcinomas are divided into cardia and non-cardia cancer based on their anatomical site.

Non-cardia cancers are brought about by chronic gastritis, inflammation of the stomach lining which can be caused by a variety of environmental factors. The pathogenesis of cardia cancer remains unclear, although two distinct etiologies have been proposed: one is associated with gastroesophageal reflux disease (GERD) and resembles esophageal adenocarcinoma, and the other is associated with $\mathrm{H}$. pylori atrophic gastritis and resembles non-cardia cancer [3].

These cancers are further histologically divided into either the intestinal (well-differentiated) or the diffuse (undifferentiated) category. The intestinal type is the more common, with the highest incidence among older males, but also carries a better prognosis [2], whereas the diffuse type affects younger people and frequently females [10].

Risk factors associated exclusively with cardia gastric cancer include obesity and GERD, while H. pylori infection, low socioeconomic status, and dietary factors are risk factors that are exclusive for non-cardia gastric cancer [11].

\section{Risk factors}

\section{Genetics}

Inherited mutations of certain genes, such as the GSTM1-null phenotype or CDH1 gene, have been found to increase the risk of stomach cancer. Loss of one of the copies of the $\mathrm{CDH} 1$ gene results in hereditary diffuse gastric cancer (HDGC), an autosomal dominantly inherited condition wherein malignant cells promulgate underneath the stomach lining and consequently metastasize. HDGC and loss of CDH1 are also associated with lobular breast cancer, prostate cancer, and colorectal cancer [12].

IL-17 and IL-10, polymorphisms of the interleukin genes especially common in Asian populations, are also associated with an elevated risk of the neoplasm. Lynch syndrome is another common hereditary disorder that increases the risk of gastric cancer $[2,12]$.

Gastric adenocarcinoma and proximal polyposis of the stomach (GAPPS) is a cancer-predisposing syndrome inherited in an autosomal dominant pattern. It has been mapped to the gene $5 q 22$, which results in the loss of the WT allele in fundic gland polyps $[12,13]$. Point mutations in the $A P C$ promoter appear to be drivers of the disorder.

Likewise, familial adenomatous polyposis (FAP), the most common form of familial intestinal gastric cancer, is an autosomal-dominant inherited predisposition to adenomatous polyps caused by germline mutations in the APC gene. Nevertheless, the lifetime risk of developing gastric cancer among FAP patients is less than 1\%, indi-

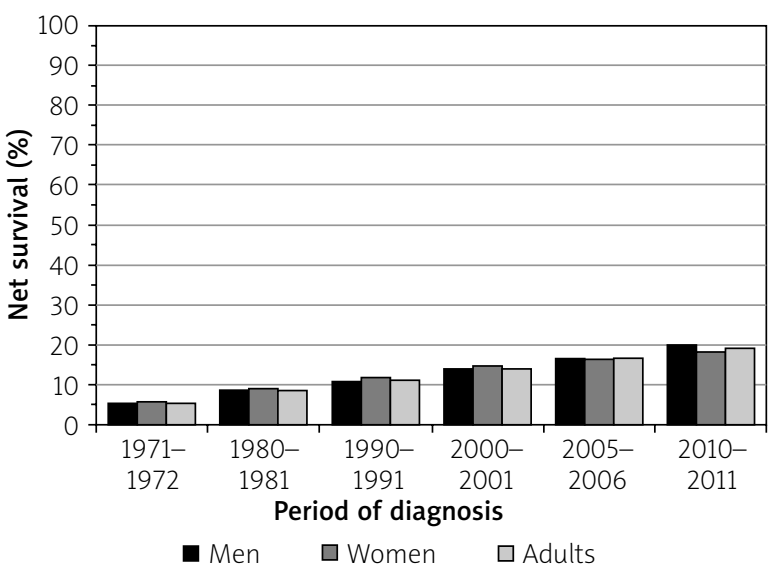

Figure 7. Bar chart of stomach cancer, age-standardized 5-year net survival, adults (aged 15-99), England and Wales, 1971-2011 (reproduced from reference [9])

cating that it requires an environmental component in order to transform the polyps into adenocarcinomas [12].

All these genetic variants have been found to contribute to $1-3 \%$ of cases [2]. A strong environmental component is believed to be the greatest factor in the regional variability of gastric cancer incidence [6].

\section{Helicobacter pylori}

The main risk factor for gastric cancer is the bacterium H. pylori. In 2005, Australian researchers Barry Marshall and Robin Warren were awarded the Nobel Prize in Medicine for the discovery of the bacterium H. pylori. Prior to their finding, lifestyle and stress were hypothesized to be the major risk factors in peptic ulcer disease. In 1985, Marshall deliberately infected himself with the bacterium to demonstrate that it caused acute gastritis. Thanks to their work, we now know that up to $80 \%$ of gastric ulcers are caused by $H$. pylori [14].

Gastritis brought about by the bacterium also elicits the majority of gastric cancers. $90 \%$ of cases of the non-cardia subtype are associated with the bacterium [3]. In fact, $H$. pylori has been found to increase the odds ratio of stomach cancer by $5.9 \times$ within ten years of infection. IL-10 and IL-17, the polymorphisms associated with gastric cancer, have been shown to interact with H. pylori infection [2].

Meanwhile, recent studies indicate that the cardia subtype has two distinct etiologies: one resembling esophageal adenocarcinoma (EAC), most commonly $H$. pylori negative, and the other resembling non-cardia cancer in etiology [3].

Genomic sequencing and post-genomic analyses of the $H$. pylori bacterium have revealed a long history of coevolution with the human host. Many have specu- 
lated that humans once had a symbiotic relationship with the bacterium and that the recent campaign for eradicating the pathogen may be misbegotten. Many nations, such as India, that have persistently high $H$. pylori infection rates do not suffer from high gastric cancer incidence. The interaction between $H$. pylori and genetics and diet may explain these discrepancies [14].

While $H$. pylori infection increases the risk of gastric cancer, it has also been shown to protect against gastro-esophageal reflux and esophageal adenocarcinoma. While the bacterium promotes corpus (non-cardia) gastritis, it is known to reduce acid secretion in the proximal (cardia) portion of the stomach, thus decreasing gastritis in the esophagus and cardia. As it reduces the risk of esophageal inflammation, $H$. pylori has also been shown to protect against cardia gastric cancer $[3,9]$. Furthermore, $H$. pylori infection has been associated with lower risks of asthma and obesity [9].

Therefore, it may be that success in eradicating H. pylori, which has decreased the incidence of non-cardia gastric cancer in the developed world, has actually increased the incidence of the cardia subtype. The United States and the United Kingdom have the highest proportion of cardia relative to non-cardia stomach cancer, possibly due to the complete absence of the $H$. pylori bacterium [15]. A recent study in Hong Kong in 63,397 subjects has shown that long-term use of proton pump inhibitors (PPIs) was associated with a 2.4-fold increase in gastric cancer risk in $\mathrm{H}$. pylori-infected subjects who had received eradication therapy. The risk of gastric cancer increases in a dose- and duration-dependent manner with the use of PPIs [16].

Advocates of the "hygiene hypothesis" further point out that exposure to a bacteria-free environment early in life, as brought about by antibiotics during the treatment of $\mathrm{H}$. pylori, increases the risk of certain autoimmune disorders later in life, while also enabling the spread of antibiotic resistance [17].

\section{Gastric ulcer}

Helicobacter pylori is the main risk factor in the association between gastric ulcer and gastric cancer [18, 19]. In a large cohort of 57,936 Swedish patients it was found that the risk of gastric cancer was almost twice the expected rate among patients with gastric ulcers and there was a $40 \%$ reduction in risk of gastric cancer among patients with duodenal ulcers [18]. Helicobacter pylori eradication in gastric ulcer patients may reduce the risk of developing gastric cancer [20].

\section{Gastroesophageal reflux disease}

Several studies have reported significant associations between GERD and cardia gastric cancer [21, 22].
Cardia gastric cancer was positively associated with both severe gastric atrophy and with frequent GERD symptoms [23]. Multiple distinct pathways of tumorigenesis exist at the esophagogastric junction, including one in which tumors arise from dysplastic intestinal metaplasia, and one potentially involving dysplasia of the cardiac-type mucosa [24]. The incidence of reflux-related cardia cancer has steadily increased when compared to $H$. pylori-related cardia cancer. Incidence rate trends in reflux-related cardia cancer and esophageal adenocarcinoma are very similar, suggesting that these two cancers share a similar etiology and pathophysiological process [25].

\section{Smoking}

Tobacco use has been shown to increase the risk of cardia gastric cancer. Tobacco has been implicated in the recent rise of cardia gastric cancer in developed nations. It is estimated that $11 \%$ of global stomach cancers, and $17 \%$ of cases in Europe, are attributable to smoking [2]. A meta-analysis of 42 studies estimated that in smokers the risk of gastric cancer was increased approximately 1.53-fold and was higher in men than in women [26]. A recent study has shown that hookah and opium use are risk factors for gastric cancer and precancerous lesions [27].

\section{Alcohol}

Alcohol use has been shown to increase the risk of gastric cancer, but the effect of the amount of alcohol consumed and gastric cancer risk has been controversial. Based on a meta-analysis of 10 studies, moderate alcohol consumption was shown to increase gastric cancer risk by $39 \%$, while heavy consumption further worsened the odds. Another meta-analysis of 44 case-control and 15 cohort studies showed a lack of association between moderate alcohol drinking and gastric cancer risk. However, a positive association of gastric cancer with heavy alcohol drinking was found [28]. Alcohol is known to irritate and erode the stomach lining, resulting in gastritis, a precursor for stomach cancer [29, 30].

\section{Chemical exposure}

Occupational exposure to dust, high-temperature particulates, and metals such as chromium VI has also been implicated in non-cardia stomach cancer. Occupations as varied as wood processing, food machine operation, rubber manufacturing, coal mining, and metal processing have been found to carry a greater risk of the neoplasm [2]. A recent meta-analysis of 40 mortality cohort studies showed that asbestos is associated 
with a moderately increased risk of stomach cancer [31]. A recent meta-analysis of 13 observational studies showed that occupational talc exposure is associated with an increased risk of stomach cancer [32]. A significant relationship has been found between occupational crystalline silica exposure and gastric cancer [33].

\section{Diet}

Ingestion of salt has been shown to increase gastritis and the carcinogenic effects of known gastric carcinogens such as $N$-methyl- $N$-nitro- $N$-nitrosoguanidine (MNNG). Salt is known to erode the mucosal barrier of the stomach, thereby leading to inflammation. Cultures whose diets are rich in salt and pickled foods, such as the Japanese, exhibit higher rates of gastric cancer. Japanese immigrants to the United States who assimilated and adopted Western foods exhibited a substantially lower rate of gastric cancer relative to those who did not assimilate their diet [29].

Preserved meats are rich in $\mathrm{N}$-nitroso compounds, which can elicit a similar effect in the body. Grain-fed red meat is especially rich in saturated fats and low in protective fats such as omega-3, which contributes to its inflammatory processes and thus increases gastric cancer risk [29].

A meta-analysis of nine prospective studies demonstrated that coffee consumption was not associated with overall gastric cancer risk. However, subgroup analysis showed that coffee consumption might be a risk factor for gastric cardia cancer [34].

Meanwhile, fruits and vegetables are rich in carotenoids, folate, phytochemicals and vitamin C, which help modulate xenobiotic metabolizing enzymes during digestion. Fruits and vegetables also contain numerous antioxidants that prevent against metabolic damage. Vitamin C, also known as ascorbic acid, is a powerful antioxidant found in high concentrations in citrus fruit. Case-control studies found that a higher intake of fruits and vegetables was associated with a $37 \%$ lower risk of gastric cancer. Non-dietary antioxidant sources such as green tea, vitamin A, C and E supplements, and selenium have seen mixed results in preventing gastric cancer [29].

\section{Obesity}

A statistical meta-analysis from around the world found that those with an excess body mass index (over $25 \mathrm{~kg} / \mathrm{m}^{2}$ ) have a 1.13 odds ratio of developing cancer. The strength of the association increased with increasing BMI. Obesity was an especially strong predisposing factor for males and non-Asians. Obesity may induce inflammation of the stomach lining via tumor necrosis factor- $\alpha$ (TNF- $\alpha$ ), interleukin-6 (IL-6), and monocyte chemoattractant protein-1 (MCP-1). Those with a diet of heavily inflammatory foods, such as a diet high in meat and low in fruits and vegetables, also have a higher risk of being obese [29, 35].

\section{Pernicious anemia}

Studies have found that those with pernicious anemia have a $6.9 \%$ chance of developing gastric cancer, particularly non-cardia cancer, which is more than five times the risk of the general population. The link between the disorders remains unknown. Chronic gastritis, as caused by $H$. pylori infection, may lead to the long-term use of PPIs, which decrease $B_{12}$ absorption and thus cause pernicious anemia. Those with pernicious anemia and gastric cancer also share many risk factors, such as alcohol consumption and smoking [36].

\section{Gastric surgery}

There is an increased risk for gastric cancer after gastric surgery [37-39]. Risk depends on the reason for the initial surgery and type of surgery [39, 40]. The Billroth II procedure (gastrojejunostomy) carries a higher risk when compared to the Billroth I procedure (gastroduodenostomy) $[39,40]$. The interval between initial gastric surgery for benign disease and the development of gastric stump carcinoma is approximately 30 years or more, compared to 12 years if surgery was done for prior gastric cancer $[39,41]$.

\section{Radiation}

Follow-up of survivors of the Hiroshima and Nagasaki atomic bombs has established radiation as a risk factor for gastric cancer [42]. Childhood cancer survivors are at 4.6 times increased risk for gastrointestinal cancers compared to the general population. The risk was highest in cancer survivors who had abdominal radiation (standardized incidence ratio (SIR) 11.2, 95\% confidence interval (CI): 7.6-16.4). However, survivors not exposed to radiation also still had a significantly increased risk (SIR: 2.4, 95\% Cl: 1.4-3.9) [43]. A study on Hodgkin's lymphoma patients who had received subdiaphragmatic radiotherapy showed dose-dependent increased risk of stomach cancer. The risk was 77 times higher in patients who received both radiation and high dose procarbazine [44]. A recent study has shown that testicular cancer (TC) patients are at $1.45 \%$ risk for stomach cancer after 30 years of TC diagnosis. Risk increases to 5.9 times in TC survivors who have received radiotherapy [45].

\section{Epstein-Barr virus (EBV)}

Approximately $5-10 \%$ of gastric carcinomas are associated with EBV [46]. A meta-analysis of 70 studies showed 


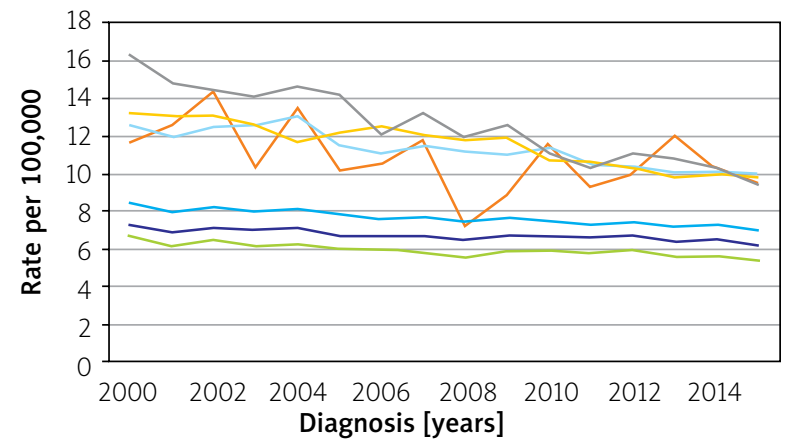

_ All races (includes Hispanic)

- American Indian/Alaska native (includes Hispanic)

_ Asian/Pacific Islander (includes Hispanic)

_ Black (includes Hispanic)

_ Hispanic (any race)

- Non-Hispanic White

— White (includes Hispanic)

Figure 8. Stomach cancer, recent trends in SEER incidence rates, 2000-2015 by race/ethnicity (reproduced from http:// seer.cancer.gov/ [60])

the EBV associated gastric cancer is two times more common in males than in females. It was also found that EBV associated gastric cancer is more common in the gastric cardia or postsurgical gastric stump area [47]. Recent studies have suggested that EBV and coinfection with $H$. pylo$r i$ increase the occurrence of gastric carcinoma [48]. The exact role of EBV in gastric carcinoma is still unclear [49].

\section{Socioeconomic status (SES)}

An increased risk of gastric cancer was associated with low SES [50]. Higher SES has been reported to be associated with a lower risk of occurrence of gastric cancer. Lower SES was associated with lower survival rates [51]. Higher education was closely associated with a reduced risk of gastric cancer, and this effect was more pronounced for cancer of the cardia when compared to non-cardia gastric cancer [52]. Higher rates of $H$. pylori infection or lower access to fresh food, fruits and vegetables may be responsible for the association of low SES and a higher risk of gastric cancer [11].

\section{Blood group}

The relation between cancer of the stomach and blood group A was first reported in 1953, by Aird et al. [53]. Numerous studies have shown that blood group A is associated with a higher risk of gastric cancer. An increase in pernicious anemia was also seen in this group [54, 55]. A large prospective population-based study in Scandinavia confirmed that blood type $A$ is associated with a higher risk of gastric cancer and that blood group 0 is associated with a higher risk of peptic ulcers [55].

\section{Sex}

Gastric cancer rates have been considerably lower in females than males $[1,56]$. A possible explanation might be that the protective effect of estrogen may lower the risk of gastric cancer in women. Other causes such as differences in diet and occupational exposure may contribute to increased gastric cancer incidence in males [57]. Gastric cancer risk is lowered by delayed menopause and increased fertility. Increased risk may be seen with anti-estrogen drugs, for example tamoxifen $[58,59]$. After menopause the incidence of gastric cancer in women has a similar pattern to that in men, but with a 10- to 15-year lag period [57].

\section{Race/ethnicity}

Data from the 2000 to 2015 Surveillance, Epidemiology, and End Results (SEER) registries in the United States show that the incidence of gastric cancer (rates per 100,000/year for both sexes in the year 2000 vs. the year 2015) among whites (7.3 vs. 6.2) is significantly lower than in Asians/Pacific Islanders (16.3 vs. 9.4), African Americans (13.2 vs. 9.8), American Indian/Alaska Native (11.7 vs. 9.5) and Hispanics (12.6 vs. 10.0) (Figure 8) [60]. A systematic review of 74 studies has shown that incidence and mortality rates from stomach cancer have decreased in the last four decades in most parts of the world, but rates have increased for some indigenous population groups. High rates of stomach cancer were observed in Indigenous Siberians, Mapuche in South America, Inuit in Arctic regions of Greenland, Canada and Alaska and Maoris in New Zealand [61].

\section{Screening}

There are significant regional differences in the incidence of gastric cancer. Japan and South Korea are countries which have national gastric cancer screening programs. Other countries where universal or population-based screening has been implemented include Venezuela and Chile. Screening for gastric cancer generally involves contrast radiography and endoscopy [62]. Other non-invasive methods used are serum pepsinogen levels, $H$. pylori serology, and serum trefoil factor 3, although their use has been controversial. Further studies are needed to define the role of these non-invasive methods of screening for gastric cancer.

Endoscopy allows direct visual examination of the gastric mucosa, and it allows for biopsy and histologic evaluation. In Japan, gastric cancer screening annually for all residents aged 40 years and older with upper gastrointestinal (UGI) series was initiated in 1983, 
and in 2015 the Japanese guidelines were updated to allow screening with either endoscopy or UGI series [63]. Similarly, in Korea, endoscopy or UGI series is recommended every two years for individuals aged 40 years and older [62]. The optimal interval for gastric cancer screening has not been established in randomized trials.

\section{Prevention}

\section{Helicobacter pylori testing and treatment}

Despite the controversy, the eradication of $H$. pylori in the developing world remains a primary goal in the fight against gastric cancer due to its strong association with non-cardia gastric cancer. Helicobacter pylori can be detected serologically, for example with an ELISA or HelicoTest or via the benchmark urea breath test (UBT). An endoscopy followed by a plate culture is a more invasive option to test for $H$. pylori infection [64]. Whether treatment is necessary for those with chronic, largely asymptomatic infections remains controversial [15].

\section{Lifestyle modifications}

Many factors other than $H$. pylori have been implicated in the incidence of gastric cancer. Daily consumption of three or more servings of alcohol and high-salt foods significantly increase the risk of gastric cancer. Processed meat was found to significantly increase the risk of non-cardia gastric cancer, while obesity was associated with a higher risk of stomach cardia cancer [2].

Other findings suggest that fruits and vegetables, especially citrus fruit such as oranges, lemons, and grapefruit, help to decrease the risk of the disease [65]. It is important to discuss with one's doctor before adding grapefruit to one's diet, as it may interact with other medications and exacerbate certain medical conditions $[29,66]$.

Diet modification is the most established form of prevention of gastric cancer. A wholesome diet rich in fruits, vegetables, whole grains, and low in alcohol, pickled foods, and processed, smoked or salted meats (especially red meat), not only lowers the risk of stomach inflammation and gastric cancer [29], but also helps prevent high blood pressure and obesity, thus decreasing one's risk for a wide array of chronic diseases [66].

Exercise is also an essential component of any weight loss program. Both are aerobic, and strength exercises are recommended to build muscle mass and lose and keep off weight [66].

Tobacco use has also been shown to increase the risk of cardia gastric cancer. Smoking is also responsible for one-third of cancer deaths in the United States [2].
Antioxidant supplements (such as vitamins A, C, and $E$ and the mineral selenium) and green tea have been suggested to reduce the risk of gastric cancer. However, studies have found that those with adequate nutrition do not further benefit from these antioxidant supplements or green tea $[29,66]$.

\section{Therapeutics and genetic testing}

Regular Aspirin, or other non-steroidal anti-inflammatory drugs (NSAIDs) such as naproxen or ibuprofen, have been shown to lower the risk of stomach cancer, as well as colon cancer. In a nationwide retrospective cohort study in Taiwan on 52,161 patients, regular NSAID use was found to be an independent protective factor for gastric cancer development (hazard ratio (HR) 0.79 for each incremental year; $p<0.001)$, especially in $H$. pylori-associated patients $(H R=0.52$ for each incremental year; $p<0.001$ ) [67]. In another meta-analysis of 24 studies by Huang et al. it was found that the use of NSAIDs is inversely associated with GC risk (risk ratio $=0.78,96 \% \mathrm{Cl}: 0.72-0.85$ ), especially for non-cardia GC risk [68]. However, NSAIDs also increase the risk of internal bleeding. Studies have not yet determined whether the benefits outweigh the costs for the general population. Therefore, NSAIDs are not recommended for the prevention of stomach cancer, though those taking them for chronic pain or as a blood thinner may benefit from a lowered risk of the neoplasm [69].

Those with a family history of stomach cancer, or who have personally had invasive lobular breast cancer before age 50, are recommended to undergo genetic testing for abnormal changes in the $\mathrm{CDH} 1$ gene, which greatly increase the risk of gastric cancer [70]. Physicians may recommend that those testing positive for this should have their stomach prophylactically removed. Lynch syndrome is another hereditary disease associated with gastric cancer [71].

\section{Conclusions}

Significant global strides have been made in the global prevention and treatment of gastric cancer. Nevertheless, the neoplasm remains the $5^{\text {th }}$ most commonly diagnosed, and the $3^{\text {rd }}$ most deadly. While H. pylori infection is the most established risk factor, eradicating the bacterium may actually increase the risk of a subtype of the disease. Instead, more focus is being placed on the interaction between diet, genetics, and $H$. pylori. Diet and lifestyle modification have been revealed to be the most effective means of preventing gastric cancer, especially in the developed world, while advances in genetic testing are enabling earlier detection and better survival. 


\section{Conflict of interest}

The authors declare no conflict of interest.

\section{References}

1. Bray F, Ferlay J, Soerjomataram I, et al. Global cancer statistics 2018: GLOBOCAN estimates of incidence and mortality worldwide for 36 cancers in 185 countries. CA Cancer J Clin 2018; 68: 394-424.

2. World Cancer Research Fund/American Institute for Cancer Research (WCRF/AICR). Continuous Update Project Report: Diet, Nutrition, Physical Activity and Stomach Cancer 2016 Revised 2018. London: World Cancer Research Fund International; 2018

3. Mukaisho K, Nakayama T, Hagiwara T, et al. Two distinct etiologies of gastric cardia adenocarcinoma: interactions among pH, Helicobacter pylori, and bile acids. Front Microbiol 2015; 6: 412.

4. Lauren P. The two histological main types of gastric carcinoma diffuse and so-called intestinal-type carcinoma. An attempt at a histo-clinical classification. Acta Pathol Microbiol Scand 1965; 64: 31-49.

5. Ferlay J, Ervik M, Lam F, et al. Global Cancer Observatory: Cancer Today. Lyon, France: International Agency for Research on Cancer. Available from: https://gco.iarc.fr/today, Accessed 09 October 2018. In. 2018.

6. Balakrishnan M, George R, Sharma A, Graham DY. Changing trends in stomach cancer throughout the world. Curr Gastroenterol Rep 2017; 19: 36.

7. Howlader NA, Krapcho M, Miller D, et al. SEER Cancer Statistics Review, 1975-2014, National Cancer Institute. Bethesda, MD, https://seer.cancer.gov/csr/1975_2014/, based on November 2016 SEER data submission, posted to the SEER web site, April 2017. 2017.

8. Wang J, Sun Y, Bertagnolli MM. Comparison of gastric cancer survival between Caucasian and Asian patients treated in the United States: results from the Surveillance Epidemiology and End Results (SEER) database. Ann Surg Oncol 2015; 22: 2965-71.

9. Cancer Research UK. https://www.cancerresearchuk.org/ health-professional/cancer-statistics/statistics-by-cancer-type/ stomach-cancer/survival\#heading-Two. Accessed October 3, 2018. In.

10. Chon HJ, Hyung WJ, Kim C, et al. Differential prognostic implications of gastric signet ring cell carcinoma: stage adjusted analysis from a single high-volume center in Asia. Ann Surg 2017; 265: 946-53.

11. Karimi P, Islami F, Anandasabapathy S, et al. Gastric cancer: descriptive epidemiology, risk factors, screening, and prevention. Cancer Epidemiol Biomarkers Prev 2014; 23: 700-13.

12. Boland CR, Yurgelun MB. Historical perspective on familial gastric cancer. Cell Mol Gastroenterol Hepatol 2017; 3: 192-200.

13. Li J, Woods SL, Healey S, et al. Point mutations in exon 1B of APC reveal gastric adenocarcinoma and proximal polyposis of the stomach as a familial adenomatous polyposis variant. Am J Hum Genet 2016; 98: 830-42.

14. Ahmed N. 23 years of the discovery of Helicobacter pylori: is the debate over? Ann Clin Microbiol Antimicrob 2005; 4: 17.
15. Graham DY. Helicobacter pylori update: gastric cancer, reliable therapy, and possible benefits. Gastroenterology 2015; 148: 719-31.

16. Peng YC, Huang LR, Lin CL, et al. Association between proton pump inhibitors use and risk of gastric cancer in patients with GERD. Gut 2018. doi: 10.1136/gutjnl-2018-316057.

17. Okada H, Kuhn C, Feillet H, Bach JF. The 'hygiene hypothesis' for autoimmune and allergic diseases: an update. Clin Exp Immunol 2010; 160: 1-9.

18. Hansson LE, Nyren O, Hsing AW, et al. The risk of stomach cancer in patients with gastric or duodenal ulcer disease. N Engl J Med 1996; 335: 242-9.

19. Molloy RM, Sonnenberg A. Relation between gastric cancer and previous peptic ulcer disease. Gut 1997; 40: 247-52.

20. Take S, Mizuno M, Ishiki K, et al. The effect of eradicating Helicobacter pylori on the development of gastric cancer in patients with peptic ulcer disease. Am J Gastroenterol 2005; 100: 1037-42.

21. Wu AH, Tseng CC, Bernstein L. Hiatal hernia, reflux symptoms, body size, and risk of esophageal and gastric adenocarcinoma. Cancer 2003; 98: 940-8.

22. Ye W, Chow WH, Lagergren J, et al. Risk of adenocarcinomas of the esophagus and gastric cardia in patients with gastroesophageal reflux diseases and after antireflux surgery. Gastroenterology 2001; 121: 1286-93.

23. Derakhshan MH, Malekzadeh R, Watabe $\mathrm{H}$, et al. Combination of gastric atrophy, reflux symptoms and histological subtype indicates two distinct aetiologies of gastric cardia cancer. Gut 2008; 57: 298-305.

24. Demicco EG, Farris AB $3^{\text {rd }}$, Baba $Y$, et al. The dichotomy in carcinogenesis of the distal esophagus and esophagogastric junction: intestinal-type vs. cardiac-type mucosa-associated adenocarcinoma. Mod Pathol 2011; 24: 1177-90.

25. Abrams JA, Gonsalves L, Neugut Al. Diverging trends in the incidence of reflux-related and Helicobacter pylori-related gastric cardia cancer. J Clin Gastroenterol 2013; 47: 322-7.

26. Ladeiras-Lopes R, Pereira AK, Nogueira A, et al. Smoking and gastric cancer: systematic review and meta-analysis of cohort studies. Cancer Causes Control 2008; 19: 689-701.

27. Sadjadi A, Derakhshan MH, Yazdanbod A, et al. Neglected role of hookah and opium in gastric carcinogenesis: a cohort study on risk factors and attributable fractions. Int J Cancer 2014; 134: 181-8.

28. Tramacere I, Negri E, Pelucchi C, et al. A meta-analysis on alcohol drinking and gastric cancer risk. Ann Oncol 2012; 23: 28-36.

29. Tsugane S, Sasazuki S. Diet and the risk of gastric cancer: review of epidemiological evidence. Gastric Cancer 2007; 10: 75-83.

30. Ma K, Baloch Z, He TT, Xia X. Alcohol consumption and gastric cancer risk: a meta-analysis. Med Sci Monit 2017; 23: 238-46.

31. Fortunato L, Rushton L. Stomach cancer and occupational exposure to asbestos: a meta-analysis of occupational cohort studies. Br J Cancer 2015; 112: 1805-15.

32. Chang CJ, Tu YK, Chen PC, Yang HY. Talc exposure and risk of stomach cancer: systematic review and meta-analysis of occupational cohort studies. J Formos Med Assoc 2018. doi: 10.1016/j.jfma.2018.07.015. 
33. Lee W, Ahn YS, Lee S, et al. Occupational exposure to crystalline silica and gastric cancer: a systematic review and meta-analysis. Occup Environ Med 2016; 73: 794-801.

34. Liu H, Hua Y, Zheng X, et al. Effect of coffee consumption on the risk of gastric cancer: a systematic review and metaanalysis of prospective cohort studies. PLoS One 2015; 10: e0128501.

35. Lin XJ, Wang CP, Liu XD, et al. Body mass index and risk of gastric cancer: a meta-analysis. Jpn J Clin Oncol 2014; 44: 783-91.

36. Kuipers EJ. Pernicious anemia, atrophic gastritis, and the risk of cancer. Clin Gastroenterol Hepatol 2015; 13: 2290-2.

37. Ahn HS, Kim JW, Yoo MW, et al. Clinicopathological features and surgical outcomes of patients with remnant gastric cancer after a distal gastrectomy. Ann Surg Oncol 2008; 15: 1632-9.

38. Komatsu S, Ichikawa D, Okamoto K, et al. Progression of remnant gastric cancer is associated with duration of follow-up following distal gastrectomy. World J Gastroenterol 2012; 18: 2832-6.

39. Takeno S, Hashimoto T, Maki K, et al. Gastric cancer arising from the remnant stomach after distal gastrectomy: a review. World J Gastroenterol 2014; 20: 13734-40.

40. Tersmette AC, Offerhaus GJ, Tersmette KW, et al. Meta-analysis of the risk of gastric stump cancer: detection of high risk patient subsets for stomach cancer after remote partial gastrectomy for benign conditions. Cancer Res 1990; 50: 6486-9.

41. Lagergren J, Lindam A, Mason RM. Gastric stump cancer after distal gastrectomy for benign gastric ulcer in a population-based study. Int J Cancer 2012; 131: E1048-52.

42. Preston DL, Ron E, Tokuoka S, et al. Solid cancer incidence in atomic bomb survivors: 1958-1998. Radiat Res 2007; 168: 1-64.

43. Henderson TO, Oeffinger KC, Whitton J, et al. Secondary gastrointestinal cancer in childhood cancer survivors: a cohort study. Ann Intern Med 2012; 156: 757-66.

44. Morton LM, Dores GM, Curtis RE, et al. Stomach cancer risk after treatment for Hodgkin lymphoma. J Clin Oncol 2013; 31: 3369-77.

45. Hauptmann M, Fossa SD, Stovall M, et al. Increased stomach cancer risk following radiotherapy for testicular cancer. $\mathrm{Br}$ J Cancer 2015; 112: 44-51.

46. Boysen T, Mohammadi M, Melbye M, et al. EBV-associated gastric carcinoma in high- and low-incidence areas for nasopharyngeal carcinoma. Br J Cancer 2009; 101: 530-3.

47. Murphy G, Pfeiffer R, Camargo MC, Rabkin CS. Meta-analysis shows that prevalence of Epstein-Barr virus-positive gastric cancer differs based on sex and anatomic location. Gastroenterology 2009; 137: 824-33.

48. Singh S, Jha HC. Status of Epstein-Barr virus coinfection with Helicobacter pylori in gastric cancer. J Oncol 2017; 2017: 3456264.

49. Cho J, Kang MS, Kim KM. Epstein-Barr virus-associated gastric carcinoma and specific features of the accompanying immune response. J Gastric Cancer 2016; 16: 1-7.

50. Uthman OA, Jadidi E, Moradi T. Socioeconomic position and incidence of gastric cancer: a systematic review and meta-analysis. J Epidemiol Community Health 2013; 67: 854-60.

51. Sun $F$, Sun $H, M o X$, et al. Increased survival rates in gastric cancer, with a narrowing gender gap and widening socioeco- nomic status gap: a period analysis from 1984 to 2013. J Gastroenterol Hepatol 2018; 33: 837-46.

52. Nagel G, Linseisen J, Boshuizen HC, et al. Socioeconomic position and the risk of gastric and oesophageal cancer in the European Prospective Investigation into Cancer and Nutrition (EPIC-EURGAST). Int J Epidemiol 2007; 36: 66-76.

53. Aird I, Bentall HH, Roberts JA. A relationship between cancer of stomach and the ABO blood groups. Br Med J 1953; 1 : 799-801.

54. Hoskins LC, Loux HA, Britten A, Zamcheck N. Distribution of $A B O$ blood groups in patients with pernicious anemia, gastric carcinoma and gastric carcinoma associated with pernicious anemia. N Engl J Med 1965; 273: 633-7.

55. Edgren G, Hjalgrim H, Rostgaard K, et al. Risk of gastric cancer and peptic ulcers in relation to ABO blood type: a cohort study. Am J Epidemiol 2010; 172: 1280-5.

56. Freedman ND, Chow WH, Gao YT, et al. Menstrual and reproductive factors and gastric cancer risk in a large prospective study of women. Gut 2007; 56: 1671-7.

57. Camargo MC, Goto Y, Zabaleta J, et al. Sex hormones, hormonal interventions, and gastric cancer risk: a meta-analysis. Cancer Epidemiol Biomarkers Prev 2012; 21: 20-38.

58. Sheh A, Ge Z, Parry NM, et al. 17beta-estradiol and tamoxifen prevent gastric cancer by modulating leukocyte recruitment and oncogenic pathways in Helicobacter pylori-infected INS-GAS male mice. Cancer Prev Res (Phila) 2011; 4: $1426-35$

59. Wang Z, Butler LM, Wu AH, et al. Reproductive factors, hormone use and gastric cancer risk: the Singapore Chinese Health Study. Int J Cancer 2016; 138: 2837-45.

60. Surveillance, Epidemiology, and End Results Program. Cancer query system: SEER Incidence Statistics (2000-2015). Available at: http://seer.cancer.gov/. Accessed October 29, 2018.

61. Arnold M, Moore SP, Hassler S, et al. The burden of stomach cancer in indigenous populations: a systematic review and global assessment. Gut 2014; 63: 64-71.

62. Kim GH, Liang PS, Bang SJ, Hwang JH. Screening and surveillance for gastric cancer in the United States: is it needed? Gastrointest Endosc 2016; 84: 18-28.

63. Hamashima C. Systematic Review G. Guideline Development Group for Gastric Cancer Screening G. Update version of the Japanese Guidelines for Gastric Cancer Screening. Jpn J Clin Oncol 2018; 48: 673-83.

64. PDQ (Physician Data Query). J Natl Cancer Inst 2017; 109.

65. Bae JM, Lee EJ, Guyatt G. Citrus fruit intake and stomach cancer risk: a quantitative systematic review. Gastric Cancer 2008; 11: 23-32.

66. Kushi LH, Doyle C, McCullough M, et al. American Cancer Society Guidelines on nutrition and physical activity for cancer prevention: reducing the risk of cancer with healthy food choices and physical activity. CA Cancer J Clin 2012; 62: 30-67.

67. Wu CY, Wu MS, Kuo KN, et al. Effective reduction of gastric cancer risk with regular use of nonsteroidal anti-inflammatory drugs in Helicobacter pylori-infected patients. J Clin Oncol 2010; 28: 2952-7.

68. Huang XZ, Chen Y, Wu J, et al. Aspirin and non-steroidal anti-inflammatory drugs use reduce gastric cancer risk: a dose-response meta-analysis. Oncotarget 2017; 8: 4781-95. 
69. Akre K, Ekstrom AM, Signorello LB, et al. Aspirin and risk for gastric cancer: a population-based case-control study in Sweden. Br J Cancer 2001; 84: 965-8.

70. Benusiglio PR, Malka D, Rouleau E, et al. CDH1 germline mutations and the hereditary diffuse gastric and lobular breast cancer syndrome: a multicentre study. J Med Genet 2013; 50: 486-9.

71. Chun N, Ford J. Genetic testing in stomach cancer, In: DeVita, Hellman, and Rosenberg's Cancer: Principles and Practice of Oncology. $10^{\text {th }}$ ed. DeVita VT, Lawrence TS, Rosenberg SA (eds.). Philadelphia, Pa, Lippincott Williams \& Wilkins 2015.

Received: 20.10 .2018

Accepted: 2.11 .2018 